\title{
Philosophiques
}

\section{La métaphysique du sens des choses}

\section{Jean Grondin}

Volume 41, numéro 2, automne 2014

URI : https://id.erudit.org/iderudit/1027223ar

DOI : https://doi.org/10.7202/1027223ar

Aller au sommaire du numéro

Éditeur(s)

Société de philosophie du Québec

ISSN

0316-2923 (imprimé)

1492-1391 (numérique)

Découvrir la revue

Citer ce document

Grondin, J. (2014). La métaphysique du sens des choses. Philosophiques, 41(2),

351-357. https://doi.org/10.7202/1027223ar d'utilisation que vous pouvez consulter en ligne.

https://apropos.erudit.org/fr/usagers/politique-dutilisation/ 


\title{
Disputatio
}

\section{La métaphysique du sens des choses}

\author{
JEAN GRONDIN
}

À la mémoire de Jean-François Mattéi (I94I-20I4)

Dans un texte célèbre, Descartes dit de la philosophie qu'elle est comme un arbre dont les racines sont la métaphysique ${ }^{1}$. L'idée banale mais anachronique que je cherche à défendre dans $D u$ sens des choses est que cela reste vrai et que, loin d'être un ballast accablant, presque honteux, dont il faudrait à tout prix se délester, comme on se plaît souvent à le claironner aujourd'hui, la métaphysique incarne un héritage que la philosophie et la pensée humaines peuvent s'approprier pour leur plus grand bénéfice. Il pourrait permettre à la philosophie de redevenir ce qu'elle a toujours été, à savoir une écoute vigilante et raisonnée du sens des choses.

La philosophie est une science particulière, si bien sûr il s'agit d'une science. Tout dépend assurément du concept de science qu'on veut lui appliquer (et il y a fort à parier que celui-ci proviendra de la métaphysique). Dans le cas de Descartes, il est clair que la philosophie correspond à l'ensemble du savoir, qui comprend la métaphysique, la physique, la mécanique, la médecine et la morale. Cette conception de la philosophie est celle qui a prévalu dans notre tradition intellectuelle jusqu'à la spécialisation des savoirs, au XIX ${ }^{e}$ siècle, qui a vu des disciplines comme la physique, la mécanique et la médecine s'émanciper de la philosophie, du tronc de la philosophie, si l'on peut en rester à l'image cartésienne de l'arbre.

La philosophie telle que nous l'entendons et la pratiquons depuis un peu moins de deux siècles a conservé, si l'on peut dire, le souci des racines, le souci métaphysique, et celui de ses applications, le souci moral. Les philosophes sont donc aujourd'hui des métaphysiciens ou des moralistes, qu'ils le sachent expressément ou non ${ }^{2}$. L'idée qui s'est un peu perdue lors de cette

1. R. Descartes, Lettre-préface à l'édition française des Principes de la philosophie, Euvres, éd. Ch. Adam \& P. Tannery, t. IX, 2: «Toute la philosophie est comme un arbre, dont les racines sont la métaphysique, le tronc est la physique, et les branches qui sortent de ce tronc sont toutes les autres sciences, qui se réduisent à trois principales, à savoir la médecine, la mécanique et la morale.»

2. Il est en effet assez remarquable d'observer que les termes de métaphysique et de morale (qui figurent dans le titre d'une des plus vénérables revues philosophiques de langue française, la Revue de métaphysique et de morale) ont été assez désertés par les philosophes euxmêmes: ils continueront de poser des questions fondamentales (du moins on l'espère), mais refuseront, sans craindre l'auto-contradiction pragmatique, de se dire métaphysiciens, d'aucuns se faisant même un point d'honneur de se dire post- ou anti-métaphysiciens. Quant au terme de morale, il a été à peu près universellement supplanté par celui d'éthique. À quoi tient ce glissement? Ce n'est pas mon sujet ici, mais j'avancerais provisoirement deux explications: $\mathrm{I}$ ) le terme 
transformation de la philosophie, c'est que la métaphysique, comprise comme la science des racines, donc des raisons ultimes, servait de fondement à l'ensemble du savoir - et par conséquent à l'idée même d'éducation - et à la bonne conduite de la vie, puisque la morale, les bonnes mœurs, en était la conséquence. Le savoir et l'être humain avaient ainsi des racines métaphysiques et un sens moral. Aujourd'hui, assez bizarrement, on semble être à la recherche d'un savoir qui serait dépourvu d'assises métaphysiques et qui, au nom de l'objectivité scientifique, serait exempt de conséquences morales. Il n'est pas surprenant que ce savoir et cette morale soient en crise. Car ce n'est pas la métaphysique elle-même qui est en crise, mais le savoir et la morale qui prétendraient vouloir s'en passer.

Je ne m'intéresserai ici qu'à la métaphysique. Si on peut la considérer comme une science, c'est parce qu'il s'agit assurément d'une "discipline ", donc de quelque chose qui s'apprend (discere) et qui nous apprend des choses. C'est à la fois une science ancienne, Parménide, Platon et Aristote en ont fondé le projet, et une science qui ne vieillit pas ou pas beaucoup. Cela se remarque chez les philosophes, nombreux dans notre tradition, qui ont fait de la métaphysique et qui se sont aussi adonnés à des enquêtes scientifiques plus spécifiques et, semble-t-il, plus empiriques. Pensons aux plus grands, Platon, Aristote, Plotin, Descartes, Leibniz, Kant, Schelling ou Hegel: si leurs travaux scientifiques de physique ou de cosmologie n'apprendront à peu près rien aux scientifiques d'aujourd'hui, qui ne leur porteront qu'un intérêt historique, et encore, il n'en va pas de même de leur métaphysique. On lira davantage la Métaphysique d'Aristote, les Méditations métaphysiques de Descartes ou la Critique de la raison pure de Kant que leurs traités du monde ou leurs essais sur les volcans lunaires. Pourquoi en est-il ainsi?

C'est que la métaphysique est moins une théorie sur le monde que sur le sens du monde, c'est-à-dire sur ce qui rend la vie digne d'être vécue et vécue avec philosophie, c'est-à-dire avec une perspective métaphysique sur le sens des choses. Ce sont ces questions, que l'on peut appeler aujourd'hui des questions de sens, qui relèvent en propre de ce que nous appelons la philosophie au sens désormais étroit du terme, la réflexion sur le sens des choses (je redis qu'en son sens large la philosophie embrassait autrefois, en gros jusqu'à Schelling et Hegel, tout l'édifice du savoir, sens qui ne survit guère que dans le beau titre de Philosophiae doctor que l'on décerne à tous les savants). L'histoire de la métaphysique est l'histoire des réponses à ces

de morale a pris un sens "moralisateur", si ce n'est janséniste, qui effraie les philosophes qui ne veulent plus «faire la morale » à personne, plus particulièrement en cette ère du «anything goes,

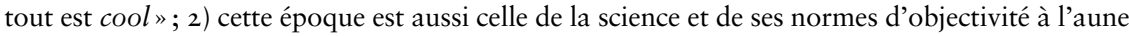
desquelles le terme d'éthique conserve une sonorité peut-être plus neutre, plus scientifique, plus clinique que celui de morale. Les éthiciens ne veulent plus défendre une morale (ce qui a pourtant toujours été leur tâche), ils se contentent d'analyser à distance et de manière soi-disant «neutre» (je n'en crois rien) les prétentions de validité dans le domaine des mœurs. 
questions et des questions que ces réponses n'ont pas manqué de susciter. C'est pourquoi l'élément de la métaphysique est celui du dialogue. La métaphysique forme ainsi un entretien de longue durée sur le sens des choses: Platon répondant à Parménide ou aux sophistes, Aristote discutant avec Platon qui regarde par-dessus son épaule dans tous ses écrits, Plotin répondant aux stoïciens, Augustin aux néoplatoniciens, Averroès à Avicenne, Thomas à Anselme, Spinoza et Leibniz à Descartes, Hegel à Kant, Heidegger à Husserl, Derrida et Gadamer à Heidegger, etc. À chaque fois c'est une position métaphysique qui s'oppose à une autre, mais dont elle est le plus souvent tributaire. On peut lire l'histoire de la philosophie comme une guerre de métaphysiques, car elle a souvent été cela et l'est encore. Mais il m'apparaît plus fécond de reconnaître que toutes ces voix font partie du canon de la métaphysique, et j'entends ici le canon en son double sens, normatif et musical. Pourquoi un platonicien devrait-il se priver des raisons d'Aristote ou un kantien de celles de Hegel? Car c'est toujours d'un dialogue de raisons qu'il s'agit, depuis que des êtres rationnels nous sommes. S'il est une chose que la métaphysique peut nous apprendre, c'est que ces raisons et ces points de vue sur le sens des choses sont multiples. Dès lors qu'ils se sont mis à réfléchir aux raisons des choses, Platon et Aristote en ont aussitôt distingué plusieurs sens. Mais tous ces sens - la cause finale, la cause idéelle, etc. - sont au service d'une seule et même quête de raison, qui caractérise la métaphysique et que la métaphysique a même introduite et fondée.

La métaphysique est un exercice de la raison. Elle nous apprend comme de raison que la raison elle-même se dit en plusieurs sens, pollachôs legetai. Cette raison est d'abord celle du monde, la raison des choses, que la métaphysique et la science ne cessent jamais de chercher, mais aussi la raison de ceux qui s'efforcent de comprendre le sens ou la raison des choses. La première phrase de la Métaphysique le dit très bien: tous les hommes aspirent à comprendre. Or qu'est-ce que comprendre sinon saisir par sa raison la raison des choses? Quelles que soient ses divisions particulières, la métaphysique comprend une ontologie (rationnelle, si l'on me passe le pléonasme) et une anthropologie qui envisage l'homme à partir de sa capacité à comprendre et sentir le sens et la raison des choses. On peut protester tant qu'on voudra contre cette vision forcément rationnelle des choses, la tirade antirationnelle suit l'histoire de la métaphysique comme son ombre, mais si l'on juge que le réel n'est pas rationnel (ou que notre raison elle-même ne l'est pas!), c'est parce que l'on juge que cette vision rationnelle des choses oublie des raisons du monde que telle ou telle métaphysique, prétendument nouvelle et iconoclaste, permet, elle, de penser. Et si l'homme n'est pas un animal rationnel, ou pas que cela, c'est parce qu'il est des raisons de l'homme dont cette perspective ne pourrait rendre raison. C'est pourquoi il est difficile d'argumenter "contre» la métaphysique: on ne peut la critiquer sans la pratiquer. On pourrait montrer sans peine que cela est vrai de la plupart des critiques sérieuses de la métaphysique, exercice auquel je ne me livrerai pas 
ici. Dans le premier chapitre de $D u$ sens des choses et dans mes autres travaux sur ces auteurs ${ }^{3}$, je m'efforce cependant de rappeler que c'est le cas des deux plus influents critiques de la métaphysique aux Temps modernes: Kant et Heidegger. Le premier ne détruit les arguments sophistiques de la raison pure spéculative que pour rendre enfin possible une métaphysique fondée sur l'ordre de la raison pratique. Quant à Heidegger, il ne déconstruit le mode de pensée métaphysique que pour que soit enfin posée la question de l'être, question que la tradition aurait systématiquement éludée selon lui, mais dont il est bien hasardeux de prétendre qu'elle n'est pas métaphysique et d'autant que cette nouvelle amorce heideggérienne de la question de l'être doit préparer une nouvelle entente du divin dans la nuit de l'oubli de l'être.

C'est plutôt un autre versant de la rationalité métaphysique que je voudrais modestement et rapidement mettre ici en évidence. C'est l'idée que la métaphysique, en découvrant la raison des choses et celle de l'homme, nous procure aussi des raisons de vivre, d'être et d'espérer. Je pense que cela est précieux à une époque qui désespère tant de l'ordre du monde et, ceci expliquant cela, de la métaphysique elle-même. La découverte inaugurale de la métaphysique - on pourrait dire son grand pari, son hypothèse, si on préfère - est que la vie humaine est sensée parce que l'ordre du monde l'est et que la raison humaine l'est tout autant, guidée qu'elle est par une attente de sens constante. Ces conceptions peuvent sonner surannées, mais, comme nous l'avons vu, en métaphysique les bonnes idées ne vieillissent pas beaucoup.

L'idée selon laquelle il y a un sens à l'ordre du monde, auquel notre raison peut espérer comprendre quelque chose, a sans doute émergé avec Platon et sa grande vision de l'eidos, de l'idée, qui incarne à la fois le fondement, la beauté et le sens des choses. Pour Platon, cette intuition relève de la pure et simple contemplation, dans tous les beaux sens du terme: dès lors que l'on s'arrête un peu pour considérer l'ordre du monde, que l'on se détache un peu du train-train des affaires courantes (ce qu'accomplit toute philosophie), on ne peut manquer d'être saisi par ce que Platon appelait l'eidos ou la beauté du monde. Eidos et beauté sont même des termes à peu près toujours interchangeables en grec, comme en latin d'ailleurs (species). Ce n'est donc pas un hasard si Platon aime tant illustrer son expérience et sa vision de l'idée à l'aide de la beauté, qui est l'affaire la plus resplendissante (ekphanestaton, Phèdre $250 \mathrm{~d}$ ), la plus irrésistible et la plus irrécusable du monde, donc la plus vraie. La seule chose dont il faille se garder ici, c'est de notre vision provinciale de la beauté qui en fait une expérience exclusivement

3. Voir à titre indicatif pour Kant: Kant et le problème de la philosophie, Paris, Vrin, I989, et "La conclusion de la Critique de la raison pure", dans Kant-Studien 8I (I990), I29I44; pour Heidegger: Introduction à la métaphysique, Presses de l'Université de Montréal, 2004, p. 329 sq.; "Pourquoi réveiller la question de l'être? ", dans J.-F. Mattéi (dir.), Heidegger et l'énigme de l'être, PUF, collection "Débats philosophiques", 2004, 43-69; "Heidegger et le défi du nominalisme ", dans Les Temps modernes, 63 (2008), $\mathrm{n}^{\circ} 650$, 235-247. 
"esthétique» ou, pire encore, purement subjective. Ce sont là des préjugés probablement indécrottables, du moins pour ce qui est de la vulgate dominante, mais contre lesquels les philosophes devraient se prémunir. Si la métaphysique est une science et quelque chose qui s'apprend, elle peut nous rappeler que la beauté désignait et désigne encore beaucoup plus que le simple plaisir esthétique ressenti par une subjectivité. Elle est d'abord et avant tout une caractéristique des choses: ce sont les choses elles-mêmes qui sont belles et elles le sont parce qu'elles affichent de la constance, de justes proportions et une certaine intelligence dans leur agencement. Contrairement à un préjugé têtu, on peut d'ailleurs à peu près toujours s'entendre sur ce qui est beau et ce qui ne l'est pas. C'est que le beau, disait Platon, resplendit à partir de lui-même. Le beau est donc avant tout une caractéristique des choses et que nous pouvons sentir de tous nos sens, et toujours avec notre intelligence.

Pour la métaphysique, la beauté est donc toujours plus qu'une affaire esthétique. Elle ne dépend pas seulement de la belle apparence des choses. La belle apparence en fait partie, bien entendu, mais les choses ont une belle apparence parce que celle-ci émane de leur finalité intrinsèque, qui fait d'elles des manifestations du Bien. C'est une idée un peu plus difficile, mais qui reste vraie: la beauté a partie liée avec la bonté. Un beau discours, la rhétorique l'a toujours su et enseigné, est toujours plus qu'un discours mélodieux et bien frappé, c'est un discours qui dit ce qui doit être dit, qui dit ce qui est et ce qui est vrai. C'est le cas des belles formules et des belles paroles, c'est-à-dire de celles qui portent et révèlent l'être des choses.

Cette beauté habite plus intimement encore l'être des choses en des sens dont nous avons un peu perdu l'évidence, mais que la métaphysique du sens des choses peut nous aider à retrouver ou à pressentir. Qu'est-ce que fondamentalement la beauté ? Nous avons vu qu'elle resplendissait, qu'elle se nichait d'abord dans les choses et qu'elle s'apparentait au beau et au vrai. Mais pourquoi en est-il ainsi ? La réponse de Platon et d'Aristote reste inspirante et transporte encore la science moderne sans qu'elle ne s'en rende toujours compte: c'est que les choses qui sont belles sont habitées d'un ordre, d'une constance et d'une intelligence qui expliquent qu'elles soient belles (et bien sûr qu'elles soient ressenties comme telles). Ce sont là, comme chacun sait, les grandes caractéristiques de l'eidos (ou de la beauté): il a quelque chose de permanent, qui préside à l'ordre des choses et qui peut être saisi par notre intelligence. Pour Platon, et en un sens pour Aristote aussi, cela est vrai de ce que nous appellerions des "qualités » ou des "espèces ", les deux ordres de réalité pour lesquels il y a des idées. Ces qualités, ce sont, par exemple, le bien, le juste et ce que la métaphysique appellera plus tard des transcendantaux: alors que les choses belles passent et se fanent, le beau, lui, demeure. Cela est aussi vrai du bien et de la vérité. Il y a là une constance dans l'ordre même des choses qui se remarque dans la régularité des espèces. Nous savons naturellement depuis Darwin que les espèces ont une origine, 
sur la longue durée, mais ce qui fascinait les Grecs, c'était un peu le contraire, savoir l'étonnante constance des espèces. Il serait trop hâtif de dire qu'elle ait été totalement remise en question par Darwin (dont le grand ouvrage sur L'origine des espèces possède un titre extraordinairement métaphysique!), parce que la permanence (relative bien sûr sub specie aeternitatis) des espèces se laisse encore remarquer et reste une supposition majeure de la science moderne: alors que les individus, les hommes et les choses particulières, passent et meurent, c'est notre lot à tous, la métaphysique l'a d'ailleurs toujours martelé, les «espèces» responsables des grands traits des êtres demeurent à peu près constantes, se transmettant de génération en génération, d'individu en individu. Le terme d'espèce est en lui-même très évocateur: c'est la traduction exacte du terme species en latin et eidos en grec. Mais en grec, comme en latin, l'espèce connote la beauté, la bonté, l'ordre et l'intelligence. C'est cet ordre et ce sens que les Grecs admiraient dans la species ou l'eidos et que l'on peut encore admirer aujourd'hui, même si l'on sait, grâce à Darwin et la science moderne, que les espèces évoluent sur le long terme.

Cette métaphysique de l'idée se maintient donc dans la science moderne, dans l'attention qu'elle prête, nécessairement et métaphysiquement, à ce qui perdure. Ce qui intéresse surtout la science, elle ne s'intéresse pas qu'à cela bien entendu, mais ce qui l'intéresse par-dessus tout, c'est la régularité des choses, dont elle cherche à faire ressortir les lois et les constantes. C'est en ce sens que le privilège de l'eidos se trouve sauvegardé: ce que la science souhaite surtout percer c'est la cause du cancer, pas seulement du cancer de tel ou tel individu. Et en nous faisant découvrir cette régularité des choses, à la faveur d'une infinité d'approches différentes, la science peut encore susciter notre émerveillement devant l'ordre des choses. J'aimerais dire qu'en cela nous pouvons être encore plus platoniciens que l'on ne pouvait l'être à l'époque de Platon: c'est que nous sommes beaucoup plus à même que lui d'apprécier le sens et la beauté des choses, grâce au raffinement de la science moderne et de ses instruments de mesure et d'observation. À mes yeux, ce motif contemplatif, sans être le seul (il y a aussi le motif économique et utilitaire, indéniable), reste l'un des plus puissants moteurs de la science, qui reste métaphysique en cela. La science continue de nous faire voir et pressentir l'ordre, la beauté et le sens des choses, celui de l'eidos ou de la species qui resplendit dans ce qui est.

C'est là le dernier aspect sur lequel j'insisterai: cet eidos en est un que l'on peut voir et contempler, grâce à notre raison, dont les Grecs ont surtout mis en valeur le versant intuitif ou contemplatif. L'eidos fait l'objet d'une "vision ». On ne peut manquer d'être sidéré par lui quand on se penche sur l'être du monde. Certes, il est de bon ton aujourd'hui de dire que l'on ne contemple pas d'essences (certains sophistes le prétendaient aussi dans l'Antiquité), seulement des «individus » — c'est là, comme chacun sait, une position métaphysique, celle du nominalisme -, mais il est frappant de constater 
que les grands métaphysiciens ou philosophes ont toujours soutenu le contraire, de Platon à Husserl et au-delà. Ils l'ont dit parce que ce que nous voyons et sentons, nous l'exprimons toujours et le faisons parler à l'aide de notions qui vont bien au-delà des cas individuels observés et qui nous permettent de cette façon d'en rendre raison. Ce que je perçois, c'est une beauté, c'est un homme, parce que cela a part à l'ordre de ce qui est beau et de ce qui est humain, ordre qui se montre justement dans ce qui apparaît.

L'une des idées directrices de la métaphysique est que notre raison et sauf erreur elle seule - a accès à cet élément de l'eidos ou de la beauté. Les deux termes (et les deux réalités) de l'eidos et de la species renvoient à l'évidence à un voir, un contempler, pour lesquels les langues anciennes ont des équivalents parfaits: les verbes eidô (dont oida est l'aoriste) ou spectare (il y a aussi speciere et ses nombreux dérivés). En effet, comment "voyons »-nous ces réalités de l'eidos? Nous les voyons bien sûr de nos yeux, cela est particulièrement évident dans le cas éblouissant de la beauté, mais il est plus juste de dire que c'est avec notre intelligence que nous les apercevons. Cette intelligence qui pénètre plus profondément l'ordre et le sens des choses distingue notre raison, notre nôेs ou notre intellectus. Que découvre, que voit ou "per-çoit» ce nô̂s? La réponse de toute la métaphysique est limpide: il entrevoit et pressent le noûs et l'intelligence des choses elles-mêmes. Toute métaphysique est l'effort de notre raison de comprendre et de communiquer cette expérience du sens des choses. En découvrant ce sens et cette raison du monde, notre raison nous donne par le fait même des raisons de vivre. La métaphysique est ainsi le bien le plus précieux de l'histoire de l'humanité et mérite autre chose que le mépris des philosophes. 